\title{
Active Surveillance for Low-risk Prostate Cancer: The European Association of Urology Position in 2018
}

\author{
Alberto Briganti ${ }^{a, *}$, Nicola Fossati ${ }^{a}$, James W.F. Catto ${ }^{b}$, Philip Cornford $^{c}$, Francesco Montorsi $^{a}$, \\ Nicolas Mottet ${ }^{d}$, Manfred Wirth ${ }^{e}$, Hendrik Van Poppel ${ }^{f}$ \\ ${ }^{a}$ Division of Oncology, Unit of Urology, URI, IRCCS Ospedale San Raffaele, Milan, Italy; ${ }^{\mathrm{b}}$ Academic Urology Unit, University of Sheffield, Sheffield, UK; ${ }^{\mathrm{c}}$ Royal \\ Liverpool and Broadgreen Hospitals NHS Trust, Liverpool, UK; ${ }^{\mathrm{d}}$ Department of Urology, University Hospital, St. Etienne, France; ${ }^{\mathrm{e}}$ Department of Urology, \\ Medical Faculty Carl Gustav Carus, Technical University of Dresden, Dresden, Germany; ${ }^{\mathrm{f}}$ Department of Urology, University Hospitals Leuven, Leuven, Belgium
}

\section{Article info}

Article history:

Accepted June 1, 2018

Associate Editor:

James Catto

\section{Keywords:}

Active surveillance

Position paper

Prostate cancer

\begin{abstract}
Active surveillance (AS) represents a well-recognized management option for many patients with low- and very low-risk prostate cancer (PCa). AS aims to reduce overtreatment whilst ensuring curative treatment for those in whom it is needed, without losing the window of curability. While long-term series have confirmed the safety of AS in carefully selected patients, this has resulted in new clinical questions. Can the inclusion criteria be expanded? Is there a role for biomarkers and multiparametric magnetic resonance imaging at diagnosis or during AS? What is the optimal follow-up schedule as well as the most meaningful trigger for definitive treatment? These questions, together with increasingly adopted heterogeneous protocols in AS, have prompted the European Association of Urology to produce a position paper corroborated by a summary of the scientific background on AS.

Patient summary: Active surveillance (AS) is becoming a widely adopted strategy for patients affected by low-risk prostate cancer. While a formal systematic review on the topic will soon be available, the European Association of Urology has produced specific statements for different open questions on AS.
\end{abstract}

(c) 2018 European Association of Urology. Published by Elsevier B.V. All rights reserved.

\footnotetext{
* Corresponding author. Division of Oncology/Unit of Urology, IRCCS Ospedale San Raffaele, VitaSalute San Raffaele University, Via Olgettina, 60, 20131 Milan, Italy. Tel. +39 022643 7286; Fax: +3902 26437298.

E-mail address: briganti.alberto@hsr.it (A. Briganti).
}

\section{Introduction}

Approximately $45 \%$ of men with screening-detected localized prostate cancer (PCa) are candidates for deferred treatment [1]. The Cluster Randomized Trial of PSA Testing for Prostate Cancer showed that a single PSA screening intervention increased the detection of low-risk PCa [2]. In this context, active surveillance (AS) represents a wellrecognized option for the initial management of selected patients with low- and very low-risk PCa. This approach is increasingly used in this setting [3,4], with the aims of reducing possible overtreatment and achieving curative treatment for those with progressive disease without losing the window of curability [5]. Patients remain under close surveillance, and treatment is prompted by predefined thresholds indicative of potentially life-threatening but still curable disease in men with adequate life expectancy. Current data supporting the role of AS are derived from ongoing prospective or retrospective cohorts. No formal randomized controlled trial is available comparing AS to standard treatment, although a randomized study of less intensive active monitoring showed no difference in overall 
survival (OS) at $10 \mathrm{yr}$ when compared to active treatment mainly in men with low- and intermediate-risk PCa [6]. The largest published AS cohort coupled with the longest followup included 993 patients with low- or intermediate-risk PCa [7]. This prospective cohort enrolled men with clinical stage $\mathrm{T} 1$ or T2a and PSA $\leq 10 \mathrm{ng} / \mathrm{ml}$, age $\leq 70 \mathrm{yr}$, and a Gleason score $\leq 6$ or age $>70 \mathrm{yr}$ with a Gleason score of $\leq 3+4$. Interestingly, men with intermediate-risk disease represented approximately $20 \%$ of the entire cohort of study. Moreover, neither multiparametric magnetic resonance imaging (mp-MRI; not available at the time of study initiation) nor extensive biopsy sampling were considered in the study. After a median follow-up of $6.4 \mathrm{yr}$, the 10 - and $15-\mathrm{yr}$ OS were $80 \%$ and $62 \%$, respectively, and cancer-specific survival (CSS) rates were $98.1 \%$ and $94.3 \%$, respectively. Radical treatment was received by $27 \%$ of this population, prompted by a PSA doubling time $<3 \mathrm{yr}(43.5 \%)$, a Gleason score progression on repeat biopsies (35\%), and patient preference (6\%). Thirty men (3\%) developed metastases during follow-up: $2 \%$ of those initially classified as Gleason 6 as compared to $9.7 \%$ if initially Gleason 7 [8]. Several other protocols have investigated AS in organ-confined disease [9], including the PRIAS study which represents the largest prospectively enrolled cohort of men initially managed with AS [10]. Although a decline in adherence has been observed in real life [11], AS showed to be safe and able to reduce the extent of overtreatment in low-risk PCa, provided accurate patient selection. Given such favorable outcomes, several studies focused on how to expand indications and to increase adherence to AS protocols [12,13]. This has contributed to an increasing number of studies and recommendations [14,15], sometimes based on single institution expertise rather than on strong, large evidence. In particular, the role of imaging and biomarkers during AS is not yet standardized and different protocols have been implemented with these approaches at different stages of AS. Some studies used mpMRI to confirm eligibility for AS [16,17], others included imaging to expand inclusion criteria for AS and to reduce misclassification by using fusion biopsies [18,19], or even to replace the key role of prostate biopsy during follow-up [20]. In addition to such recent implementations, there are still considerable variations among studies regarding patient selection, follow-up schedule, the use of confirmatory or repeat biopsy and what should trigger active treatment. Moreover, existing guidelines regarding AS for PCa vary widely [21]. These differences not only make comparison between these studies difficult but also contribute to highly heterogeneous protocols for AS, which is confusing to both physicians and patients. All these reasons have prompted the European Association of Urology (EAU) to produce a position paper on AS, as done previously for other topics [22-24].

\section{Selection criteria for AS}

\subsection{Statement}

Include all men with low-risk PCa for AS using a standardized prospective protocol. Men with longer life expectancy (ie, $>20 \mathrm{yr}$ ) should be properly counseled about the lack of very long-term data of AS.

\subsection{Scientific background}

There are several long-term prospective AS cohorts that have been reported, with different inclusion criteria and different protocols [25-29]. These different selection criteria included men fit for curative treatment and a life expectancy of at least $10 \mathrm{yr}$ with low/very low-risk disease defined on a combination of PSA, clinical stage, and number of positive cores (Table 1). Stricter criteria are better at defining clinically insignificant disease but exclude many men with indolent cancer. Less stringent criteria (ie, enrolment of men with low-risk PCa regardless of the extent of cancer at biopsy) also resulted in excellent CSS outcomes.

The two largest and most mature prospective AS cohorts include men with low-risk PCa managed at Sunnybrook Health Sciences Centre and Johns Hopkins University $[7,30]$. Data from the Hopkin's series showed that only two low-risk men (0.15\%) died from PCa at $15 \mathrm{yr}$, whilst 30\% of men died from competing causes [30]. Similarly, in the Canadian prospective cohort, the 10 -yr overall CSS rate was $98 \%$ [7]. In both series, the number of cores involved with cancer and the greatest percentage of cores involved at first biopsy were significant predictors of both PCa reclassification to higher grade and increased tumor extent at rebiopsy. However, they were not associated with disease progression over time. These results match historical data derived from men receiving nonstandardized conservative management of low-risk PCa where the rate of 10-yr CSS was $92 \%[31]$.

Data from the ProtecT trial are also supportive of a favorable outcome of conservatively managed patients with localized disease. In the study by Hamdy et al [6], low- and intermediate-risk men initially managed with active monitoring did not show any increased risk of cancerspecific death compared with men randomized to active treatment. Interestingly, such outcomes were reached using a less stringent follow-up when compared to current AS protocols.

Similar results have been reported by the PIVOT trial, even though with significantly higher rates of overall mortality at $10 \mathrm{yr}$ mainly due to poor patient selection [32]. Taken together, this data supports the indication to expand AS to all men with low-risk disease, regardless of the extent of $\mathrm{PCa}$ at initial biopsy. Moreover, the recent introduction of mp-MRI may also add value in correctly expanding indications of AS to all low-risk men. Initial negative mp-MRI at the time of AS initiation has been indeed shown to reduce the number of misclassified cancers [33]. Low-risk men with negative mp-MRI may have indeed favorable outcome on AS, regardless of the extent of low-grade cancer at biopsy [20,34,35]. Moreover, in case of a positive mp-MRI, the number of positive cores may lose its possible prognostic effect on the rate of misclassification. Several positive cores detected at the level 
Table 1 - Active surveillance inclusion criteria

\begin{tabular}{|c|c|c|c|c|c|c|c|c|}
\hline Criteria & $\begin{array}{l}\text { Date of } \\
\text { initiation }\end{array}$ & Note & T stage & $\begin{array}{l}\text { Gleason } \\
\text { score }\end{array}$ & $\begin{array}{l}\text { PSA threshold } \\
(\mathrm{ng} / \mathrm{ml})\end{array}$ & $\begin{array}{l}\text { No. of } \\
\text { cores }\end{array}$ & PSAD & $\begin{array}{c}\text { Core } \\
\text { involvement } \\
(\%)\end{array}$ \\
\hline $\begin{array}{l}\text { Goteborg } \\
\text { (Godtman Eur Urol 2016) }\end{array}$ & Jan 1995 & \multicolumn{7}{|c|}{$\begin{array}{l}\text { AS was defined as no treatment within } 6 \text { mo from diagnosis. The most common reason for choosing AS was } \\
\text { presumed low-risk PCa, but deferring treatment could also be due to comorbidities or patient preference. }\end{array}$} \\
\hline John Hopkins & Jan 1995 & very low-risk & $\mathrm{T} 1 \mathrm{c}$ & $\leq 6$ & - & Max 2 & $<0.15$ & $<50$ \\
\hline (Tosoian JCO 2015) & & older patients & $\leq \mathrm{T} 2 \mathrm{a}$ & $\leq 6$ & 101 & - & - & - \\
\hline $\begin{array}{l}\text { Miami } \\
\text { (Soloway Eur Urol 2010) }\end{array}$ & Feb 1992 & & $\mathrm{~T} 1-\mathrm{T} 2$ & $\leq 6$ & 15 & Max 2 & - & $<20$ \\
\hline $\begin{array}{l}\text { MSKCC } \\
\text { (Adamy J Urol 2011) }\end{array}$ & Sep 1997 & & $\mathrm{~T} 1-\mathrm{T} 2 \mathrm{a}$ & $\leq 6$ & 10 & Max 3 & - & $<50$ \\
\hline PRIAS & 2006 & & $\leq \mathrm{T} 2 \mathrm{c}$ & $\leq 6$ & 10 & Max 2 & $<0.20$ & - \\
\hline (Bokhorst Eur Urol 2016) & 2012 & if aged $\geq 70 \mathrm{yr}$ & $\leq \mathrm{T} 2 \mathrm{c}$ & $\leq 3+4$ & 10 & Max 2 & $<0.20$ & $<10$ \\
\hline & 2015 & $\begin{array}{l}\text { More than two cores } \\
\text { can be positive if an } \\
\text { MRI, including targeted } \\
\text { biopsies, is done at } \\
\text { inclusion } \\
\text { - If saturation biopsies } \\
\text { are done ( } 20 \text { cores), } \\
\text { 15\% of cores can be } \\
\text { positive with a } \\
\text { maximum of } 4\end{array}$ & $\leq \mathrm{T} 2 \mathrm{c}$ & $\leq 6$ & 10 & - & $<0.20$ & - \\
\hline $\begin{array}{l}\text { Royal Marsden } \\
\text { (Selvadurai Eur Urol 2013) }\end{array}$ & Mar 2002 & $\begin{array}{l}\text { Overall } \\
\text { if aged }>65 \mathrm{yr}\end{array}$ & $\mathrm{T} 1-\mathrm{T} 2$ & $\begin{array}{l}\leq 6 \\
\leq 3+4\end{array}$ & 15 & $\begin{array}{l}<50 \% \\
\text { cores }\end{array}$ & - & - \\
\hline Sunnybrook & From Nov & - All low-risk & - & $\leq 6$ & 10 & - & - & - \\
\hline (Klotz JCO 2015) & $\begin{array}{l}1995 \text { to Dec } 1999 \\
\text { From Jan } 2000\end{array}$ & $\begin{array}{l}\text { - Age }>70 \mathrm{yr} \\
\text { - All low-risk } \\
\text { - Favorable } \\
\text { intermediate-risk with } \\
\text { life expectancy }<10 \mathrm{yr}\end{array}$ & - & $\begin{array}{l}\leq 3+4 \\
\leq 6 \\
\leq 3+4\end{array}$ & $\begin{array}{l}15 \\
10 \\
20\end{array}$ & - & - & - \\
\hline UCSF (Welty J Urol 2015) & 1990 & & $\mathrm{~T} 1-\mathrm{T} 2 \mathrm{a}$ & $\leq 6$ & 10 & $\begin{array}{l}<33 \% \\
\text { cores }\end{array}$ & - & $<50$ \\
\hline
\end{tabular}

of the same prostate lesion targeted by mp-MRI-guided biopsies may not be indicative of higher rates of cancer progression at subsequent biopsy. Therefore, patients may be diagnosed with several positive cores taken from the index lesion (IL) but still have good outcomes on AS.

For all these reasons, patients fulfilling low-risk criteria should be counseled about AS as initial management of PCa highlighting pros and cons of this approach as compared to active treatment. Patients should be aware of the lack of prospective randomized evidence comparing formal AS with any curative treatment but should also be re-assured about the excellent outcome of AS and the absence of solid evidence supporting any negative oncological effect in delaying active treatment in this patient group [7,30]. However, patients should also be aware of the lack of prospective data on the outcome of AS beyond 15-20 yr from diagnosis. Therefore, men with longer life expectancy should be properly counseled about the lack of very longterm data of AS. By contrast, young patients with long life expectancy may be those who have more to gain from delaying radical therapy for many years. Therefore, after proper counseling, they could still be initially managed with AS-provided accurate biopsy. This is due to the association between the number of cores taken and the risk of biopsy reclassification [36]. Such recommendations may change when very long-term data of AS become available.

\section{The role of pathologist and the need for pathological review}

\subsection{Statement}

Report the number of cores submitted for pathological assessment and the number of positive cores per side. Report the linear percentage or length (in $\mathrm{mm}$ ) of involvement by carcinoma in the single core with the greatest amount of tumor. Finally, consider central pathological review of biopsy specimens when commencing long-term conservative treatments.

\subsection{Scientific background}

Accurate histopathologic assessment at biopsy is crucial for the selection of men suitable for AS. According to a recent consensus meeting, the pathologic factors to be considered include Gleason grade, tumor extent measurements, histologic subtype of carcinoma, presence of perineural invasion, and involvement of peri-prostatic fat [37]. In particular, pathological variants that should likely exclude a man from 
AS include prostatic adenocarcinoma with predominant ductal carcinoma histology (including pure intraductal carcinoma with the exception of high-grade prostatic intraepithelial neoplasia-like ductal adenocarcinoma), sarcomatoid and small cell carcinoma, as well as cribiform pattern.

Moreover, several detailed information should be given at initial biopsy, including the number and the fraction of positive cores, the linear percentage of carcinoma in each site (core), the linear percentage of carcinoma in the core with greatest amount of tumor, the overall linear percentage of carcinoma across all sites (cores), the linear millimeters of carcinoma in each site (cores), the linear millimeters of carcinoma in the core with greatest amount of tumor, and the total linear millimeters of carcinoma across all sites (cores). Although the role of some of these measurements is still unclear in terms of patient outcome on AS, accurate biopsy assessment is important to decrease the rates of PCa misclassification and to improve patient counseling. Indeed, some of these measurements are strictly related to misclassification although their impact on AS outcomes in terms of cancer control is still unknown.

Moreover, the role of these measurements not only at initial biopsy abut also at confirmatory/follow-up biopsy is still unclear. In addition, it is yet to be determined whether such extensive assessment maintains prognostic significance also at targeted biopsies, given the increased ability to better target possible foci of PCa under MRI guidance. Therefore, although detailed biopsy characteristics are useful for improved PCa risk assessment, their role on long-term hard clinical endpoint in men with low-risk disease is still to be elucidated. Finally, although no solid evidence supports the role of second-opinion pathology on the re-assessment of prostate biopsies, it may be useful for an accurate selection of patients on AS, especially commencing long-term conservative treatments [38].

\section{Use and timing of MRI in active surveillance}

mp-MRI can be used to detect clinically significant PCa in men on AS in order to improve patient selection and to optimize cancer diagnosis and timing for intervention. This can be done in three settings during AS:

1. mp-MRI at the time of initial diagnosis.

2. mp-MRI before confirmatory biopsy.

3. mp-MRI during follow-up.

\section{1. $\quad$ mp-MRI at the time of initial diagnosis}

\subsubsection{Statement}

In patients without mp-MRI prior to initial biopsy who are diagnosed with low-risk PCa suitable for AS, mp-MRI is recommended to rule out significant PCa missed by the initial biopsy. In men diagnosed with low-risk PCa after mpMRI, all the aforementioned inclusion criteria apply. In case of initial biopsy done under guidance of mp-MRI, both targeted and systematic biopsies are recommended.

\subsubsection{Scientific background}

Interest in the role of mp-MRI in the diagnosis of PCa was heightened following the publication of the PROMIS [39] and PRECISION study [40]. The PROMIS study suggested that mpMRI might be able to avoid under grading in $18 \%$ of cases whilst avoid one in four biopsies. However, concerns about the reproducibility of this data have prevented a firm recommendation for pre-biopsy MRI. Recently, the first large, prospective randomized trial (PRECISION study) supporting the role of pre-first biopsy mp-MRI in reducing the number of low risk PCa detected while increasing the diagnosis of clinically significant disease has been published [40]. If additional evidence will support the role of triage mpMRI, the number of low-risk men suitable for AS will inevitably decrease and new AS protocols for mp-MRIdetected intermediate-risk PCa will be likely available in the future. Recently, the role of mp-MRI for patients enrolled in AS has been addressed by a systematic review [33]. A lesion on MRI suspicious for PCa (namely, positive MRI) is seen in two-thirds of men otherwise suitable for AS. A positive MRI makes the identification of clinically significant disease at repeat biopsy more likely, especially when biopsies are targeted to suspicious MRI lesions [41]. Moreover, radical prostatectomy data showed that a positive MRI is more likely to be associated with upgrading (Gleason score $>3+3$ ) than a negative MRI ( $43 \%$ vs $27 \%$ ) [42]. Therefore, mp-MRI is currently encouraged in men after an initial diagnosis of lowrisk PCa at ultrasound-guided biopsy to rule out the presence of misdiagnosed higher-grade disease at initial biopsy.

\section{2. mp-MRI before confirmatory biopsy}

\subsubsection{Statement}

mp-MRI should be performed before a confirmatory biopsy done within 12 mo after initial diagnosis. At confirmatory biopsy, include systematic and targeted biopsies.

\subsubsection{Scientific background}

At confirmatory biopsy in men who did not receive a previous mp-MRI, the rate of PCa reclassification by using targeted biopsies ranges from $2 \%$ to $22 \%$ [33,43-46]. The added value of mp-MRI for surveillance/repeat biopsies (hence, more than $1 \mathrm{yr}$ following the confirmatory biopsy assessment) has not been evaluated yet. However, combined data of confirmatory and surveillance repeat biopsies showed a PCa reclassification rate due to targeted biopsies of $2-14 \%[19,47,48]$. The concordance of systematic and targeted biopsies at confirmatory biopsies is approximately $80 \%$. Nevertheless, omitting systematic biopsies may induce a misclassification rate of $3-13 \%$ [33,43-45]. Therefore, systematic biopsy should always be performed, even in the presence of a normal mp-MRI. Targeted biopsies of suspicious lesions on mp-MRI are mainly performed for Prostate Image Reporting and Data System (PIRADS) $\geq 3$ lesions. Although increased rates of reclassification occur in PIRADS 4 and 5 lesions, a substantial proportion of PIRADS 3 lesions show reclassification following targeted biopsies $[43,44]$, thereby confirming the significance to biopsy PIRADS $\geq 3$ lesions during AS. 


\section{3. mp-MRI during AS}

\subsubsection{Statement}

At present, there are no robust published data to support the use of, or timing of, mp-MRI instead of repeat standard biopsy to detect progression over time. Therefore, at present mp-MRI should not solely replace repeat biopsy during AS. Moreover, use of mp-MRI prior to any follow-up biopsy is not supported by any strong evidence. However, it might be of interest to better target mp-MRI-detected lesions. In case of negative mp-MRI during follow-up, men should undergo systematic biopsies. In case of low-risk PCa detected at targeted and/or systematic biopsy despite a positive mpMRI, patients should continue AS provided fulfillment of all previously listed inclusion criteria.

\subsubsection{Scientific background}

Although mp-MRI may be considered important in monitoring men on AS, robust data on the use of repeat mp-MRI instead of repeat biopsy in AS is currently lacking. Data supportive of a possible shift from repeat biopsy to repeat mp-MRI only are coming from single Institution series with high expertise on imaging [49,50]. Therefore, these results should be interpreted within the setting of an experimental approach without strong, prospective, multi-institutional validation. Prospective studies with clear definitions of radiological significance and progression are needed before this approach can be adopted. Indeed, it has also been shown that mp-MRI may miss up to $15 \%$ of clinically significant cancer during follow-up of AS [51]. Therefore, at present, mp-MRI cannot replace systematic, repeat biopsy in the follow-up of AS. The real pre-requisites for repeat use of mp-MRI during AS is proper quality image acquisition and expert readership. Scans deemed of insufficient quality should be repeated before biopsy as done in a recently published, prospective protocol [39].

\section{Optimal follow-up schedule}

\subsection{Statement}

The follow-up strategy still relies mainly on clinical and biopsy assessment. It is based on serial digital rectal examination (at least once a year), PSA (at least once every $6 \mathrm{mo}$ ), and repeated biopsy (at a minimum interval of 3-5 yr). mp-MRI cannot be used as a stand-alone tool to trigger follow-up biopsies.

\subsection{Scientific background}

Follow-up schedules of the largest AS series are reported in Table 2. Based on two single-center studies [50,52], not all patients with progression/reclassification at biopsy had radiological progression and vice versa. Therefore, mp-MRI cannot be used as a stand-alone tool to trigger follow-up biopsies; however, efforts are being made to define and standardize radiological progression during AS [53]. Moreover, radiological progression alone is not a trigger "per se" for any active intervention. However, at follow-up biopsy,
mp-MRI can be useful to decrease the number of cancers reclassified as high grade and thus suitable for active treatment. Nevertheless, whether this leads to better cancer control rates over time is yet to be determined. Results from ongoing randomized trials, The Study of Active Monitoring in Sweden [54], and the SPCG17: Prostate Cancer Active Surveillance Trigger Trial (NCT02914873), will show relevant data regarding the optimal follow-up schedule.

\section{Expanding indications for AS: The EAU perspective}

\subsection{Inclusion of "low-volume" Gleason score 3+4}

\subsubsection{Statement}

Despite the evidence that some men with Gleason $3+4$ may have favorable final pathology and good outcomes on AS, it is clear that these men have an increased risk of clinical progression and the development of metastasis when monitored using an AS protocol. If men with limited Gleason $3+4$ at biopsy decide to undergo AS, this should be done within a standardized protocol where patients are correctly informed about the lack of robust data and the increased risk of progression.

\subsubsection{Scientific background}

Although most published data on AS has focused on men with very low- and low-risk PCa, patients and practitioners continue to question selection criteria for this approach. Some have advocated expanding these to include men with intermediate-risk features. Three groups, Sunnybrook (Toronto, Canada), Royal Marsden (London, UK), and UCSF (San Francisco, CA, USA) included intermediate-risk patients in prospectively collected AS cohorts.

In the Sunnybrook surveillance cohort, 22\% were intermediate risk, with Gleason $<3+4$ and/or PSA 10-20 $\mathrm{ng} / \mathrm{ml}$. One third of these men were aged $<70 \mathrm{yr}$. The analysis of this group has provided some important observations. Despite close monitoring and intervention for evidence of risk progression, the 15-yr PCa metastasisfree survival was $84 \%$ (3.7 times higher in the intermediatethan in low-risk group) [55]. This increase in risk was associated almost solely with the presence of Gleason 7 cancer at initial diagnosis. The hazards ratio for $15-\mathrm{yr}$ PCa mortality for Gleason $3+4$ compared to $3+3$ was 4.0 ; for Gleason $4+3$, it was 10.5 . Amongst this group, the actuarial 15-yr metastasis rate was at least 20\% [8]. Importantly, a PSA above 10 did not confer a significant increased risk of metastasis in the absence of Gleason pattern 4 PCa.

The Royal Marsden group also took an inclusive approach to eligibility. In their cohort, $7 \%$ had Gleason 7 disease. The presence of Gleason 7 disease was associated with a 3.4 greater likelihood of finding "adverse pathology" on subsequent biopsy [56]. Of the two PCa deaths in their cohort, one was in a patient with Gleason 7 at baseline. A limitation of many reports of conservative management of intermediaterisk disease is the relatively short duration of follow-up.

UCSF [57] reported no difference in biopsy progression or treatment rates between low and intermediate CAPRA score 


\begin{tabular}{lcccccc}
\hline Studies & $N$ & $\begin{array}{c}\text { Median } \\
\text { follow-up (yr) }\end{array}$ & $\begin{array}{c}\text { Overall } \\
\text { survival }\end{array}$ & $\begin{array}{c}\text { Cancer-specific } \\
\text { survival }\end{array}$ & $\begin{array}{c}\text { Reclassification } \\
\text { rate }\end{array}$ & $\begin{array}{c}\text { Curative } \\
\text { intervention } \\
\text { rate }\end{array}$ \\
\hline Goteborg & 474 & $8.0 \mathrm{yr}$ & $10 \mathrm{yr}: 80 \%$ & $10 \mathrm{yr}: 99.5 \%$ & - & $10 \mathrm{yr}: 53 \%$ \\
[Godtman Eur Urol 2016] & & & $15 \mathrm{yr}: 51 \%$ & $15 \mathrm{yr}: 96.0 \%$ & & $15 \mathrm{yr} 66 \%$
\end{tabular}

- The cohort was followed with PSA tests every 3-6 mo. However, this interval could be extended to $12 \mathrm{mo}$ in older patients with stable disease.

- An early rebiopsy was typically performed in all men with a small amount $(<2 \mathrm{~mm})$ of cancer at the diagnostic biopsy to confirm that they were suitable candidates for

\begin{tabular}{|c|c|c|c|c|c|c|}
\hline $\begin{array}{l}\text { John Hopkins } \\
\text { [Tosoian JCO 2015] }\end{array}$ & 1298 & $5.0 \mathrm{yr}$ & $\begin{array}{l}10 \mathrm{yr}: 93 \% \\
15 \mathrm{yr}: 69 \%\end{array}$ & $\begin{array}{l}10 \text { yr: } 99.9 \% \\
15 \text { yr: } 99.9 \%\end{array}$ & $\begin{array}{l}10 \mathrm{yr}: 26 \% \\
15 \mathrm{yr}: 31 \%\end{array}$ & $\begin{array}{l}10 \text { yr: } 50 \% \\
15 \text { yr: } 57 \%\end{array}$ \\
\hline $\begin{array}{l}\text { Miami } \\
\text { [Soloway Eur Urol 2010] }\end{array}$ & 230 & $3.6 \mathrm{yr}$ & - & 3 yr: $100 \%$ & - & $\begin{array}{l}33 \mathrm{mo}: \\
14 \%\end{array}$ \\
\hline $\begin{array}{l}\text { MSKCC } \\
\text { [Adamy J Urol 2011] }\end{array}$ & 238 & $1.8 \mathrm{yr}$ & NR & NR & $\begin{array}{l}2 \text { yr: } 20 \% \\
5 \text { yr: } 40 \%\end{array}$ & - \\
\hline $\begin{array}{l}\text { PRIAS } \\
\text { [Bokhorst Eur Urol 2016] }\end{array}$ & 5302 & NR & $\begin{array}{l}5 \text { yr: } 97 \% \\
10 \text { yr: } 89 \%\end{array}$ & $\begin{array}{l}5 \mathrm{yr}:>99 \% \\
10 \mathrm{yr}:>99 \%\end{array}$ & $\begin{array}{l}5 \text { yr: } 34 \% \\
10 \text { yr: } 41 \%\end{array}$ & $\begin{array}{l}5 \text { yr: } 52 \% \\
10 \text { yr: } 73 \%\end{array}$ \\
\hline $\begin{array}{l}\text { Royal Marsden } \\
\text { [Selvadurai Eur } \\
\text { Urol 2013] }\end{array}$ & 471 & $5.7 \mathrm{yr}$ & 8 yr: $91 \%$ & 8 yr: $98 \%$ & 5 yr: $22 \%$ & 5 yr: $30 \%$ \\
\hline $\begin{array}{l}\text { Sunnybrook } \\
\text { [Klotz JCO 2015] }\end{array}$ & 993 & $6.4 \mathrm{yr}$ & $\begin{array}{l}10 \text { yr: } 80 \% \\
15 \text { yr: } 62 \%\end{array}$ & $\begin{array}{l}10 \text { yr: } 98.1 \% \\
15 \text { yr: } 94.3 \%\end{array}$ & - & $\begin{array}{l}10 \mathrm{yr}: 36 \% \\
15 \mathrm{yr}: 45 \%\end{array}$ \\
\hline $\begin{array}{l}\text { UCSF } \\
\text { [Welty J Urol 2015] }\end{array}$ & 810 & $5.0 \mathrm{yr}$ & 5 yr: $98 \%$ & 5 yr: $100 \%$ & 5 yr: $60 \%$ & 5 yr: $40 \%$ \\
\hline
\end{tabular}

- Further rebiopsies were recommended if disease progression (PSA and/or T-stage progression) was suspected, and every $2-3 \mathrm{yr}$ in men with stable disease. - Semiannual PSA measurement for PSA decrease/increase - Semint

- Annual 12-14-core biopsy for most men

- Curative intervention was recommended for disease reclassification, defined as biopsy findings no longer meeting the inclusion criteria

Each AS patient was followed every 3-4 mo with a PSA and RE for the first $2 \mathrm{yr}$, then every 6 mo.

- In addition, after 2000, a laterally directed and peripherally targeted TRUS biopsy of 10-12 cores was performed 9-12 mo after the initial visit (the first rebiopsy), and then every year or earlier if there was a dramatic rise in PSA or a change after RE. - After initiating our AS program patients were generally followed semiannually with DRE, free and total PSA measurements, and a review of general health and urinary symptoms.

- Biopsy was routinely recommended within 12-18 mo of starting AS and subsequently repeated every $2-3$ yr or as prompted by a change in DRE or a sustained PSA increase. Treatment was recommended when the patient no longer met study eligibility criteria during follow-up.

- In the first $2 \mathrm{yr}$ of follow-up, a PSA test is scheduled every $3 \mathrm{mo}$, and a DRE is scheduled every 6 mo.

- Thereafter, PSA is measured every $6 \mathrm{mo}$, and DRE is performed once yearly. - Standard repeat biopsies are scheduled 1, 4, 7, and $10 \mathrm{yr}$ after diagnosis and subsequently every $5 \mathrm{yr}$. Yearly biopsies are only recommended if PSA doubling time (PSA DT) is between 0 and $10 \mathrm{yr}$.

- Clinical assessment with DRE and serum PSA levels taken at 3-mo intervals in the 1 st year, 4-mo intervals in the 2nd year, and at 6-mo intervals thereafter - Transrectal ultrasound-guided prostate biopsy was performed after 18-24 mo on surveillance and every $2 \mathrm{yr}$ thereafter.

- PSA was performed every $3 \mathrm{mo}$ for $2 \mathrm{yr}$ and then every $6 \mathrm{mo}$ in stable patients. A confirmatory biopsy was performed within $12 \mathrm{mo}$ of the initial biopsy and then every 3-4 yr until the patient turned $80 \mathrm{yr}$ old.

- Recommended monitoring includes quarterly PSA testing, semiannual transrectal ultrasound and annual biopsy.

- The first surveillance (ie, confirmatory) biopsy is recommended within 12 mo of diagnostic biopsy. Subsequent surveillance biopsies are recommended every 12-24 mo based on clinical risk. 
patients, albeit with 47 mo of follow-up. It is plausible from these low mortality rate differences that some patients with intermediate-risk features are surveillance candidates. In particular, these are patients with low volume of disease and older age [31,58]. Another yet retrospective assessment of AS for intermediate-risk PCa is derived from the National Prostate Cancer Register of Sweden [59] that compared outcomes of 4163 intermediate-risk patients who were managed with AS (23\%), radical prostatectomy (52\%), and radiation therapy (25\%). Amongst patients with intermediate-risk disease, the PCa mortality was $5.2 \%$ in the AS group, $3.4 \%$ in the radical prostatectomy group, and $3.8 \%$ in the radiation therapy group. These figures are similar to those reported in the Sunnybrook cohort.

Finally, a study from four centers of the European Randomized Study of Screening for Prostate Cancer included 50 men affected by Gleason score 7 and initially managed expectantly. Despite median follow-up was $2.6 \mathrm{yr}$, the 6-yr CSS (nine patients at risk) was $100 \%$ and sharply contrasted with the $68 \%$ OS [60].

\section{Patient age and AS: Are there any lower or upper age cut-off?}

\subsection{Young patients}

\subsubsection{Statement}

After appropriate risk stratification, patients should be counseled that outcomes of AS appear similar between younger and older patients, albeit that younger men need longer follow-up. Therefore, young age is not a preclusion from AS. However, young patient $(<55 \mathrm{yr})$ with long life expectancy (ie, $>20 \mathrm{yr}$ ) need to be counseled regarding the lack of robust data at very long-term follow-up, although they may also have more to gain from delaying radical therapy for many years.

\subsubsection{Scientific background}

Overtreatment of low-risk PCa is a major concern because of the associated side effects heavily influencing quality of life, particularly in younger men. However, inclusion of younger patients in AS programs is still a matter of controversy given their very long life expectancy and the ultimate need for definitive therapy. Despite this concern, it has been reported that outcome of younger men on AS are even better than their older counterpart, mainly due to lower rates of upgrading. A recent study focused on a large cohort of patients $(n=1433)$ provided evidence regarding the validity of AS for young patients [61]. Younger age was independently associated with a lower risk of Gleason score upgrading. The biopsy-based Gleason score upgrade-free rate was $73 \%$ at $3 \mathrm{yr}$ and $55 \%$ at $5 \mathrm{yr}$ for men aged $\leq 60 \mathrm{yr}$, compared to $64 \%$ and $48 \%$, respectively, for men aged $\geq 60 \mathrm{yr}$ $(p<0.01)$. Consequently, younger remained longer on AS. In addition, there was no association between younger age and risk of definitive treatment or biochemical recurrence after delayed radical prostatectomy. The study showed that after appropriate risk stratification, patients may be counseled that outcomes are similar among younger and older patients at intermediate follow-up. Therefore, younger patients should be advised that intermediate-term outcomes are not worse, but that very long-term follow-up is still needed. Moreover, younger patients should also be counseled about the need for longer periods of adherence to standardized AS protocols as well as about a possible impact of multiple biopsies on functional outcomes at least after radical prostatectomy although this is still a matter of debate $[62,63]$.

\subsection{Elderly patients}

\subsubsection{Statement}

Individual health status and comorbidity, rather than age itself, should be used to decide treatment for an individual's PCa. A life expectancy of $10 \mathrm{yr}$ is most commonly used as threshold for deciding on AS rather than on watchful waiting, although this can be questioned as all the available data suggest the risk of harm in $<15 \mathrm{yr}$ for these patients is minimal.

\subsubsection{Scientific background}

Evaluation of health status and life expectancy is fundamental in clinical decision-making on treatment of PCa. In localized disease, $>10 \mathrm{yr}$ of life expectancy is considered mandatory for any benefit from local treatment [15]. The International Society of Geriatric Oncology PCa working group recommends that treatment for senior adults should be based on a systematic evaluation of health status using the Geriatric 8 (G8) screening tool [64]. Healthy patients with a G8 score $>14$ or frail patients with reversible impairment after resolution of their geriatric problems should receive the same treatment as younger patients. Disabled patients with irreversible impairment should receive adapted treatment. Patients who are too ill should receive only palliative treatment. Patients with a G8 score $\leq 14$ should undergo a full geriatric evaluation as this score is associated with 3-yr mortality, assessing comorbidity, nutritional status, and cognitive and physical functions to determine if the impairment is reversible [65].

\section{Race and AS}

\subsection{Statement}

Given the lack of prospective data on AS outcomes stratified according to race, patient race should not modify eligibility to and follow-up during AS.

\subsection{Scientific background}

Previous studies have reported higher rate of occult coexistent cancer in low-risk African-American patients as well as higher rates of biochemical recurrence after surgery $[66,67]$. However, one large recent study of the SEARCH database including $40 \%$ African-American men with low-risk cancer showed no difference in pathological upstaging or biochemical recurrence, reinforcing the use of surveillance in these men [68]. No strong data exist suggesting different 
biological profile of different patient races in men suitable for AS. Moreover, no strong data support any indication for genetic mutation assessment in men either candidate or on AS, regardless of patient race. African-American men should be informed that they are more likely to harbor anterior and potentially higher-grade tumors that may have implications for enrolment into AS programs [69,70].

\section{Use of biomarkers in AS}

\subsection{Statement}

Biological markers, including PSA isoforms, urine PCA3, TMPRSS2-ERG, 4K score, or GPS appear promising to guide care of men with AS. However, they have not yet been prospectively robustly tested in the AS setting. Therefore, while waiting for further data and even though some men may benefit for assessment of these biomarkers, their use cannot be currently routinely recommended in AS.

\subsection{Scientific background}

9.2.1. [-2]proPSA and Prostate Health Index

[-2]proPSA and Prostate Health Index, measured at diagnosis or during AS, have discriminatory power for the endpoint unfavorable histopathological reclassification according to the Epstein criteria [71-73]. Data are missing on its additional value compared with classical parameters. In terms of clinical utility, the discriminatory power is similar among studies [74].

\subsubsection{PCA3 and TMPRSS2:ERG score}

PCA3 and TMPRSS2:ERG seem to stratify the risk of having aggressive cancer as defined by tumor volume or Gleason score. However, they did not show any added or discriminatory and independent value compared with PSA for prediction of Gleason score $\geq 7$ tumors [75].

\subsubsection{Kscore}

The 4Kpanel has been significantly associated with reclassification at the first surveillance biopsy, providing incremental value over routine clinical information, and the $4 \mathrm{~K}$ model performed significantly better than the base model. However, the 4Kpanel did not add predictive value to a PSA clinical model for biopsy decision-making for men at subsequent surveillance biopsies [76].

\subsubsection{Genomic markers}

Three genetic tissue assays (Genomic classifier, Genomic prostate score, and Cell cycle progression) have been approved by the FDA for men with PCa. Genomic classifier is a score calculated from a gene-expression microarray analysis of 22 genes on formalin-fixed paraffin-embedded prostate tumor tissue, ranges between 0 and 1 , and classifies patients into three risk categories [77]. Genomic prostate score is a biopsy-based 17-gene score that improves prediction of the presence or absence of adverse pathology and may help men with PCa make more informed decisions between AS and immediate treatment [78]. Cell cycle progression test is a genomic test designed to help provide an individualized assessment of the risk of disease progression in patients with low- and intermediate-risk PCa [79]. While these genomic markers may be used to optimize patient classification and management, none of these tests has yet been specifically validated as providing substantial benefit in the AS population. However, the "sweet spot" for molecular biomarkers is the otherwise favorable-risk patient with small amount of pattern 4 (Grade Group 2) on biopsy who prefers a conservative approach if possible. A low score on a validated molecular assay provides a reassurance about the safety of nonintervention.

The inclusion of biomarkers into multivariable models may help improving the ability to predict patient outcomes on AS. A recent study evaluating nomograms based on clinical variables alone found indeed modest ability of these models to predict cancer and clinical progression during AS [80].

\section{Patient distress during AS: The role of psycho- oncologist}

\subsection{Statement}

Patient distress should not necessarily trigger radical treatment. Men with favorable-risk PCa suitable for AS should be properly reassured about the safety of this approach. Therefore, psycho-oncological support in men on AS is encouraged to minimize patient distress and optimize adherence to AS.

\subsection{Scientific background}

Patient preference accounts for approximately $6 \%$ of reasons for discontinuing surveillance [7]. Moreover, patient preference is related to non-adherence, which in the end may lead to discontinuation. To reduce distress frequently experienced by men diagnosed with PCa and their partners, the following steps are needed: (1) improved community and medical awareness of AS, (2) consistent information about the safety of AS which does not decrease the rates of curability of active treatment when delivered, (3) appropriate counseling on when radical treatment is required, and (4) consistent, unbiased information on treatment options, prognostic indicators, and side effects. Finally, regularly updated decisional support information/aids incorporating men's values are necessary $[81,82]$.

\section{When to end AS}

\subsection{Statement}

The decision to deliver active treatment should be based on the deterioration (ie, disease progression) of the prior inclusion criteria (Table 1 ). A worsening in mp-MRI features such as increased volume of the index lesion, appearance of new lesions or increased PIRADS score at a given lesion do not represent "per se" trigger for intervention. Rather, these parameters should be used to optimize timing, targeting, and extent of biopsies. When the life expectancy decreases, 
the decision to remain on AS despite increasing in PCa grade should be individualized. However, in case of a decrease in life expectancy to $<10 \mathrm{yr}$, a switch from AS to a watchful waiting protocol is indicated.

\subsection{Scientific background}

Need for active treatment is based on a change in grade, or in the extent of cancer involvement at biopsy or in local stage $(>\mathrm{T} 2)$. These criteria are well recognized in all published cohorts [83]. A PSA change (especially a PSA doubling time of $<3 \mathrm{yr}$ ) is a less powerful indicator to change management based on its weak link with grade progression $[84,85]$. Similarly, it seems to be a less power predictor of disease reclassification and progression [10]. Active treatment may also be delivered upon patient request. This occurs in around 10\% of patients on AS [7]. While mp-MRI may show worsening of PIRADS score or appearance or new lesions or increasing in volume of the index lesion, these parameters should not be used per se to trigger intervention. Imaging features should instead be always complemented with targeted and systematic biopsies, which may confirm local disease progression. Even at follow-up biopsies, targeted biopsy should be always performed with systematic sampling, given the association between PIRADS score of the IL and the risk of missing significant PCa outside the IL $[51,86]$. Finally, since $25-40 \%$ of men remain on AS at $10 \mathrm{yr}$ of follow-up [7,30], the decision to continue on AS in these men with decreasing life expectancy despite increasing in PCa grade should be individualized. In men with a decreased life expectancy to $<10 \mathrm{yr}$ based on careful patient assessment including change in comorbidity profile over time, a switch from AS to watchful and waiting is indicated.

\section{Conclusions}

AS should be considered for all men with low-risk PCa, fit for curative treatment, and willing to adhere to AS protocols, including younger men who are provided accurate assessment to exclude misclassification of higher-grade disease. However, men with very long life expectancy should be counseled about the lack of very long-term data of AS. Consider central pathological review of the biopsy specimens in patients within AS programs, especially when recommendations for reporting criteria are not respected. While use of biomarkers cannot be routinely recommended in AS, mp-MRI can be used to optimize the diagnosis of clinically significant $\mathrm{PCa}$ in order to improve patient selection and reduce the rates of misclassification. The strongest evidence available is in favor of the use of mp-MRI prior to confirmatory biopsy. Although the role of imaging is increasing even during AS, the follow-up strategy still relies mainly on clinical and biopsy assessment. It is based on serial digital rectal examination (at least once/year), PSA (at least once every $6 \mathrm{mo}$ ), and repeated biopsy (at a minimum interval of 3-5 yr). Currently, mp-MRI should not be used as a stand-alone tool to trigger follow-up biopsies or active intervention. The decision to deliver active treatment should be based on the deterioration (ie, disease progression) of the prior inclusion criteria used at AS initiation. Finally, the position of the EAU will be updated after a formal consensus meeting and a formal systematic review on AS which will be available in early 2019. While waiting for such consensus, the current statements represent the formal position of the EAU on AS and they complement the EAU guidelines.

Author contributions: Alberto Briganti had full access to all the data in the study and takes responsibility for the integrity of the data and the accuracy of the data analysis.

Study concept and design: Briganti, Fossati, Catto, Cornford, Montorsi, Mottet, Wirth, Poppel.

Acquisition of data: Briganti, Fossati, Catto, Cornford, Montorsi, Mottet, Wirth, Poppel.

Analysis and interpretation of data: Briganti, Fossati, Catto, Cornford, Montorsi, Mottet, Wirth, Poppel.

Drafting of the manuscript: Briganti, Fossati, Catto, Cornford, Montorsi, Mottet, Wirth, Poppel.

Critical revision of the manuscript for important intellectual content: Catto, Cornford, Mottet.

Statistical analysis: None.

Obtaining funding: None.

Administrative, technical, or material support: None.

Supervision: Montorsi, Wirth, Poppel.

Other (specify): None.

Financial disclosures: Alberto Briganti certifies that all conflicts of interest, including specific financial interests and relationships and affiliations relevant to the subject matter or materials discussed in the manuscript (eg, employment/affiliation, grants or funding, consultancies, honoraria, stock ownership or options, expert testimony, royalties, or patents filed, received, or pending), are the following: None.

Funding/Support and role of the sponsor: None.

\section{References}

[1] Albertsen PC. Observational studies and the natural history of screen-detected prostate cancer. Curr Opin Urol 2015;25:232-7.

[2] Martin RM, Donovan JL, Turner EL, et al. Effect of a low-intensity PSA-based screening intervention on prostate cancer mortality: the CAP randomized clinical trial. Jama 2018;319:883-95.

[3] Cooperberg MR, Carroll PR. Trends in management for patients with localized prostate cancer, 1990-2013. Jama 2015;314:80-2.

[4] Loeb S, Folkvaljon Y, Curnyn C, Robinson D, Bratt O, Stattin P. Uptake of active surveillance for very-low-risk prostate cancer in Sweden. JAMA Oncol 2017;3:1393-8.

[5] Welty CJ, Cooperberg MR, Carroll PR. Meaningful end points and outcomes in men on active surveillance for early-stage prostate cancer. Curr Opin Urol 2014;24:288-92.

[6] Hamdy FC, Donovan JL, Lane JA, et al. 10-year outcomes after monitoring, surgery, or radiotherapy for localized prostate cancer. N Engl J Med 2016;375:1415-24.

[7] Klotz L, Vesprini D, Sethukavalan P, et al. Long-term follow-up of a large active surveillance cohort of patients with prostate cancer. J Clin Oncol 2015;33:272-7.

[8] Yamamoto T, Musunuru B, Vesprini D, et al. Metastatic prostate cancer in men initially treated with active surveillance. J Urol 2016;195:1409-14.

[9] Thomsen FB, Brasso K, Klotz LH, Røder MA, Berg KD, Iversen P. Active surveillance for clinically localized prostate cancer-a systematic review. J Surg Oncol 2014;109:830-5. 
[10] Bokhorst LP, Valdagni R, Rannikko A, et al. A decade of active surveillance in the PRIAS study: an update and evaluation of the criteria used to recommend a switch to active treatment. Eur Urol 2016;70:954-60.

[11] Bokhorst LP, Alberts AR, Rannikko A, et al. Compliance rates with the Prostate Cancer Research International Active Surveillance (PRIAS) protocol and disease reclassification in noncompliers. Eur Urol 2015;68:814-21.

[12] Klotz L. Active surveillance for intermediate risk prostate cancer. Curr Urol Rep 2017;18:80.

[13] Dall'Era MA, Klotz L. Active surveillance for intermediate-risk prostate cancer. Prostate Cancer Prostatic Dis 2017;20:1-6.

[14] Chen RC, Rumble RB, Loblaw DA, et al. Active surveillance for the management of localized prostate cancer (Cancer Care Ontario Guideline): American Society of Clinical Oncology Clinical Practice Guideline Endorsement. J Clin Oncol 2016;34:2182-90.

[15] EAU-ESTRO-ESUR-SIOG Guidelines on Prostate Cancer. 2018.

[16] Park BH, Jeon HG, Choo SH, et al. Role of multiparametric 3.0-Tesla magnetic resonance imaging in patients with prostate cancer eligible for active surveillance. BJU Int 2014;113:864-70.

[17] Henderson DR, de Souza NM, Thomas K, et al. Nine-year follow-up for a study of diffusion-weighted magnetic resonance imaging in a prospective prostate cancer active surveillance cohort. Eur Urol 2016;69:1028-33.

[18] Volkin D, Turkbey B, Hoang AN, et al. Multiparametric magnetic resonance imaging (MRI) and subsequent MRI/ultrasonography fusion-guided biopsy increase the detection of anteriorly located prostate cancers. BJU Int 2014;114:E43-9.

[19] Da Rosa MR, Milot L, Sugar L, et al. A prospective comparison of MRIUS fused targeted biopsy versus systematic ultrasound-guided biopsy for detecting clinically significant prostate cancer in patients on active surveillance. J Magn Reson Imaging 2015;41:220-5.

[20] Margel D, Yap SA, Lawrentschuk N, et al. Impact of multiparametric endorectal coil prostate magnetic resonance imaging on disease reclassification among active surveillance candidates: a prospective cohort study. J Urol 2012;187:1247-52.

[21] Bruinsma SM, Bangma CH, Carroll PR, et al. Active surveillance for prostate cancer: a narrative review of clinical guidelines. Nat Rev Urol 2016;13:151-67.

[22] Abrahamsson P-A, Artibani W, Chapple CR, Wirth M. European Association of Urology position statement on screening for prostate cancer. Eur Urol 2009;56:270-1.

[23] Mirone V, Debruyne F, Dohle G, et al. European Association of Urology position statement on the role of the urologist in the management of male hypogonadism and testosterone therapy. Eur Urol 2017;72:164-7.

[24] van der Poel HG, van den Bergh RCN, Briers E, et al. Focal therapy in primary localised prostate cancer: The European Association of Urology Position in 2018. Eur Urol 2018;74:84-91.

[25] Reese AC, Landis P, Han M, Epstein JI, Carter HB. Expanded criteria to identify men eligible for active surveillance of low risk prostate cancer at Johns Hopkins: a preliminary analysis. J Urol 2013;190:2033-8.

[26] Bul M, Zhu X, Valdagni R, et al. Active surveillance for low-risk prostate cancer worldwide: the PRIAS study. Eur Urol 2013;63:597603.

[27] Berglund RK, Masterson TA, Vora KC, Eggener SE, Eastham JA, Guillonneau BD. Pathological upgrading and up staging with immediate repeat biopsy in patients eligible for active surveillance. J Urol 2008;180, 1964-7-discussion1967-8.

[28] Soloway MS, Soloway CT, Eldefrawy A, Acosta K, Kava B, Manoharan M. Careful selection and close monitoring of low-risk prostate cancer patients on active surveillance minimizes the need for treatment. Eur Urol 2010;58:831-5.
[29] Porten SP, Whitson JM, Cowan JE, et al. Changes in prostate cancer grade on serial biopsy in men undergoing active surveillance. J Clin Oncol 2011;29:2795-800.

[30] Tosoian JJ, Mamawala M, Epstein JI, et al. Intermediate and longerterm outcomes from a prospective active-surveillance program for favorable-risk prostate cancer. J Clin Oncol 2015;33:3379-85.

[31] Lu-Yao GL, Albertsen PC, Moore DF, et al. Outcomes of localized prostate cancer following conservative management. Jama 2009;302:1202-9.

[32] Wilt TJ, Jones KM, Barry MJ, et al. Follow-up of prostatectomy versus observation for early prostate cancer. N Engl J Med 2017;377:132-42.

[33] Schoots IG, Petrides N, Giganti F, et al. Magnetic resonance imaging in active surveillance of prostate cancer: a systematic review. Eur Urol 2015;67:627-36.

[34] Sanguedolce F, Petralia G, Sokhi H, et al. Baseline Multiparametric MRI for selection of prostate cancer patients suitable for active surveillance: which features matter? Clin Genitourin Cancer 2018;16:155-6.

[35] Mullins JK, Bonekamp D, Landis P, et al. Multiparametric magnetic resonance imaging findings in men with low-risk prostate cancer followed using active surveillance. BJU Int 2013;111:1037-45.

[36] Mamawala MM, Rao K, Landis P, et al. Risk prediction tool for grade re-classification in men with favourable-risk prostate cancer on active surveillance. BJU Int 2017;120:25-31.

[37] Amin MB, Lin DW, Gore JL, et al. The critical role of the pathologist in determining eligibility for active surveillance as a management option in patients with prostate cancer: consensus statement with recommendations supported by the College of American Pathologists, International Society of Urological Pathology, Association of Directors of Anatomic and Surgical Pathology, the New Zealand Society of Pathologists, and the Prostate Cancer Foundation. Arch Pathol Lab Med 2014;138:1387-405.

[38] Barqawi AB, Turcanu R, Gamito EJ, et al. The value of second-opinion pathology diagnoses on prostate biopsies from patients referred for management of prostate cancer. Int J Clin Exp Pathol 2011;4:468-75.

[39] Ahmed HU, El-Shater Bosaily A, Brown LC, et al. Diagnostic accuracy of multi-parametric MRI and TRUS biopsy in prostate cancer (PROMIS): a paired validating confirmatory study. Lancet 2017;389:815-22.

[40] Kasivisvanathan V, Rannikko AS, Borghi M, et al. MRI-targeted or standard biopsy for prostate-cancer diagnosis. N Engl J Med 2018;378:1767-77.

[41] Siddiqui MM, Rais-Bahrami S, Truong H, et al. Magnetic resonance imaging/ultrasound-fusion biopsy significantly upgrades prostate cancer versus systematic 12-core transrectal ultrasound biopsy. Eur Urol 2013;64:713-9.

[42] Arsov C, Becker N, Rabenalt R, et al. The use of targeted MR-guided prostate biopsy reduces the risk of Gleason upgrading on radical prostatectomy. J Cancer Res Clin Oncol 2015;141:2061-8.

[43] Recabal P, Assel M, Sjoberg DD, et al. The efficacy of multiparametric magnetic resonance imaging and magnetic resonance imaging targeted biopsy in risk classification for patients with prostate cancer on active surveillance. J Urol 2016;196:374-81.

[44] Pessoa RR, Viana PC, Mattedi RL, et al. Value of 3-Tesla multiparametric magnetic resonance imaging and targeted biopsy for improved risk stratification in patients considered for active surveillance. BJU Int 2016;1:2.

[45] Kamrava M, Kishan AU, Margolis DJ, et al. Multiparametric magnetic resonance imaging for prostate cancer improves Gleason score assessment in favorable risk prostate cancer. Pract Radiat Oncol 2015;5:411-6.

[46] Ouzzane A, Renard-Penna R, Marliere F, et al. Magnetic resonance imaging targeted biopsy improves selection of patients considered for active surveillance for clinically low risk prostate cancer based on systematic biopsies. J Urol 2015;194:350-6. 
[47] Ma TM, Tosoian JJ, Schaeffer EM, et al. The role of multiparametric magnetic resonance imaging/ultrasound fusion biopsy in active surveillance. Eur Urol 2017;71:174-80.

[48] Abdi H, Pourmalek F, Zargar H, et al. Multiparametric magnetic resonance imaging enhances detection of significant tumor in patients on active surveillance for prostate cancer. Urology 2015;85:423-8.

[49] Nassiri N, Margolis DJ, Natarajan S, et al. Targeted biopsy to detect Gleason score upgrading during active surveillance for men with low versus intermediate risk prostate cancer. J Urol 2017;197:632-9.

[50] Walton-Diaz A, Shakir NA, George AK, et al. Use of serial multiparametric magnetic resonance imaging in the management of patients with prostate cancer on active surveillance. Urol Oncol 2015;33, 202.e1-7.

[51] Schulman AA, Sze C, Tsivian E, Gupta RT, Moul JW, Polascik TJ. The contemporary role of multiparametric magnetic resonance imaging in active surveillance for prostate cancer. Curr Urol Rep 2017;18:52.

[52] Felker ER, Wu J, Natarajan S, et al. Serial magnetic resonance imaging in active surveillance of prostate cancer: incremental value. J Urol 2016;195:1421-7.

[53] Moore CM, Giganti F, Albertsen P, et al. Reporting magnetic resonance imaging in men on active surveillance for prostate cancer: The PRECISE recommendations-a report of a European School of Oncology task force. Eur Urol 2017;71:648-55.

[54] Bratt O, Carlsson S, Holmberg E, et al. The Study of Active Monitoring in Sweden (SAMS): a randomized study comparing two different follow-up schedules for active surveillance of low-risk prostate cancer. Scand J Urol 2013;47:347-55.

[55] Musunuru HB, Yamamoto T, Klotz L, et al. Active surveillance for intermediate risk prostate cancer: survival outcomes in the Sunnybrook experience. J Urol 2016;196:1651-8.

[56] Selvadurai ED, Singhera M, Thomas K, et al. Medium-term outcomes of active surveillance for localised prostate cancer. Eur Urol 2013;64:981-7.

[57] Cooperberg MR, Cowan JE, Hilton JF, et al. Outcomes of active surveillance for men with intermediate-risk prostate cancer. J Clin Oncol 2011;29:228-34.

[58] Newcomb LF, Thompson IM, Boyer HD, et al. Outcomes of active surveillance for clinically localized prostate cancer in the prospective, multi-institutional canary PASS cohort. J Urol 2016;195:313-20.

[59] Stattin P, Holmberg E, Johansson J-E, et al. Outcomes in localized prostate cancer: National Prostate Cancer Register of Sweden follow-up study. J Natl Cancer Inst 2010;102:950-8.

[60] van den Bergh RCN, Roemeling S, Roobol MJ, et al. Gleason score 7 screen-detected prostate cancers initially managed expectantly: outcomes in 50 men. BJU Int 2009;103:1472-7.

[61] Leapman MS, Cowan JE, Nguyen HG, et al. Active surveillance in younger men with prostate cancer. J Clin Oncol 2017;35:1898-904.

[62] Rosenbaum CM, Mandel P, Tennstedt P, et al. Effect of repeat prostate biopsies on functional outcomes after radical prostatectomy. Urol Oncol 2018;36, 91.e17-22.

[63] Anderson CB, Tin AL, Sjoberg DD, et al. Association between number of prostate biopsies and patient-reported functional outcomes after radical prostatectomy: implications for active surveillance protocols. BJU Int 2016;117:E46-51.

[64] Droz J-P, Albrand G, Gillessen S, et al. Management of prostate cancer in elderly patients: recommendations of a task force of the international society of geriatric oncology. Eur Urol 2017;72:521-31.

[65] Bellera CA, Rainfray M, Mathoulin-Pélissier S, et al. Screening older cancer patients: first evaluation of the G-8 geriatric screening tool. Ann Oncol 2012;23:2166-72.

[66] Sundi D, Faisal FA, Trock BJ, et al. Reclassification rates are higher among African American men than Caucasians on active surveillance. Urology 2015;85:155-60.
[67] Jalloh M, Myers F, Cowan JE, Carroll PR, Cooperberg MR. Racial variation in prostate cancer upgrading and upstaging among men with low-risk clinical characteristics. Eur Urol 2015;67:451-7.

[68] Leapman MS, Freedland SJ, Aronson WJ, et al. Pathological and biochemical outcomes among African-American and Caucasian men with low risk prostate cancer in the SEARCH database: implications for active surveillance candidacy. J Urol 2016;196:1408-14.

[69] Sundi D, Kryvenko ON, Carter HB, Ross AE, Epstein JI, Schaeffer EM. Pathological examination of radical prostatectomy specimens in men with very low risk disease at biopsy reveals distinct zonal distribution of cancer in black American men. J Urol 2014;191:60-7.

[70] Kongnyuy M, Sidana A, George AK, et al. The significance of anterior prostate lesions on multiparametric magnetic resonance imaging in African-American men. Urol Oncol 2016;34, 254.e15-21.

[71] Isharwal S, Makarov DV, Sokoll LJ, et al. ProPSA and diagnostic biopsy tissue DNA content combination improves accuracy to predict need for prostate cancer treatment among men enrolled in an active surveillance program. Urology 2011;77, 763.e1-6.

[72] Tosoian JJ, Loeb S, Feng Z, et al. The impact of baseline [-2]proPSArelated indices on the prediction of pathological reclassification at 1 year during active surveillance for low-risk prostate cancer: the Japanese multicenter study cohort. J Urol 2012;188:1131-6.

[73] Hirama H, Sugimoto M, Ito K, Shiraishi T, Kakehi Y. The impact of baseline [-2]proPSA-related indices on the prediction of pathological reclassification at 1 year during active surveillance for low-risk prostate cancer: the Japanese multicenter study cohort. J Cancer Res Clin Oncol 2013;140:257-63.

[74] Lamy P-J, Allory Y, Gauchez A-S, et al. Prognostic biomarkers used for localised prostate cancer management: a systematic review. Eur Urol Focus. In press. https://doi.org/10.1016/j.euf.2017.02.017.

[75] Lin DW, Newcomb LF, Brown EC, et al. Urinary TMPRSS2:ERG and PCA3 in an active surveillance cohort: results from a baseline analysis in the Canary Prostate Active Surveillance Study. Clin Cancer Res 2013;19:2442-50.

[76] Lin DW, Newcomb LF, Brown MD, et al. Evaluating the four Kallikrein panel of the 4 Kscore for prediction of high-grade prostate cancer in men in the Canary prostate active surveillance study. Eur Urol 2017;72:448-54.

[77] Klein EA, Haddad Z, Yousefi K, et al. Decipher genomic classifier measured on prostate biopsy predicts metastasis risk. Urology 2016;90:148-52.

[78] Klein EA, Cooperberg MR, Magi-Galluzzi C, et al. A 17-gene assay to predict prostate cancer aggressiveness in the context of Gleason grade heterogeneity, tumor multifocality, and biopsy undersampling. Eur Urol 2014;66:550-60.

[79] Shore ND, Kella N, Moran B, et al. Impact of the cell cycle progression test on physician and patient treatment selection for localized prostate cancer. J Urol 2016;195:612-8.

[80] Wang S-Y, Cowan JE, Cary KC, Chan JM, Carroll PR, Cooperberg MR. Limited ability of existing nomograms to predict outcomes in men undergoing active surveillance for prostate cancer. BJU Int 2014;114:E18-24.

[81] O'Callaghan C, Dryden T, Hyatt A, et al. 'What is this active surveillance thing?.' Men's and partners' reactions to treatment decision making for prostate cancer when active surveillance is the recommended treatment option. Psycho-Oncology 2014;23:1391-8.

[82] Ruane-McAteer E, Porter S, O'Sullivan JM, Santin O, Prue G. Active surveillance for favorable-risk prostate cancer: Is there a greater psychological impact than previously thought? A systematic, mixed studies literature review. Psycho-Oncology 2017;26:1411-21.

[83] Mottet N, Bellmunt J, Bolla M, et al. EAU-ESTRO-SIOG Guidelines on prostate cancer. part 1: screening, diagnosis, and local treatment with curative intent. Eur Urol 2017;71:618-29. 
[84] Ross AE, Loeb S, Landis P, et al. Prostate-specific antigen kinetics during follow-up are an unreliable trigger for intervention in a prostate cancer surveillance program. J Clin Oncol 2010;28:2810-6.

[85] Thomsen FB, Brasso K, Berg KD, et al. Association between PSA kinetics and cancer-specific mortality in patients with localised prostate cancer: analysis of the placebo arm of the SPCG-6 study. Ann Oncol 2016;27:460-6.

[86] Stable A, Dell'Oglio P, De Cobelli F, et al. MP53-16 The more you see, the more you miss: Association between PI-RADS score of the index lesion and multifocal, clinically significant prostate cancer. Eur Urol Oncology 2018;199:e710. http://dx.doi.org/10.1016/j.euo.2018.01.002.

17-18 May 2019, Heilbronn, Germany

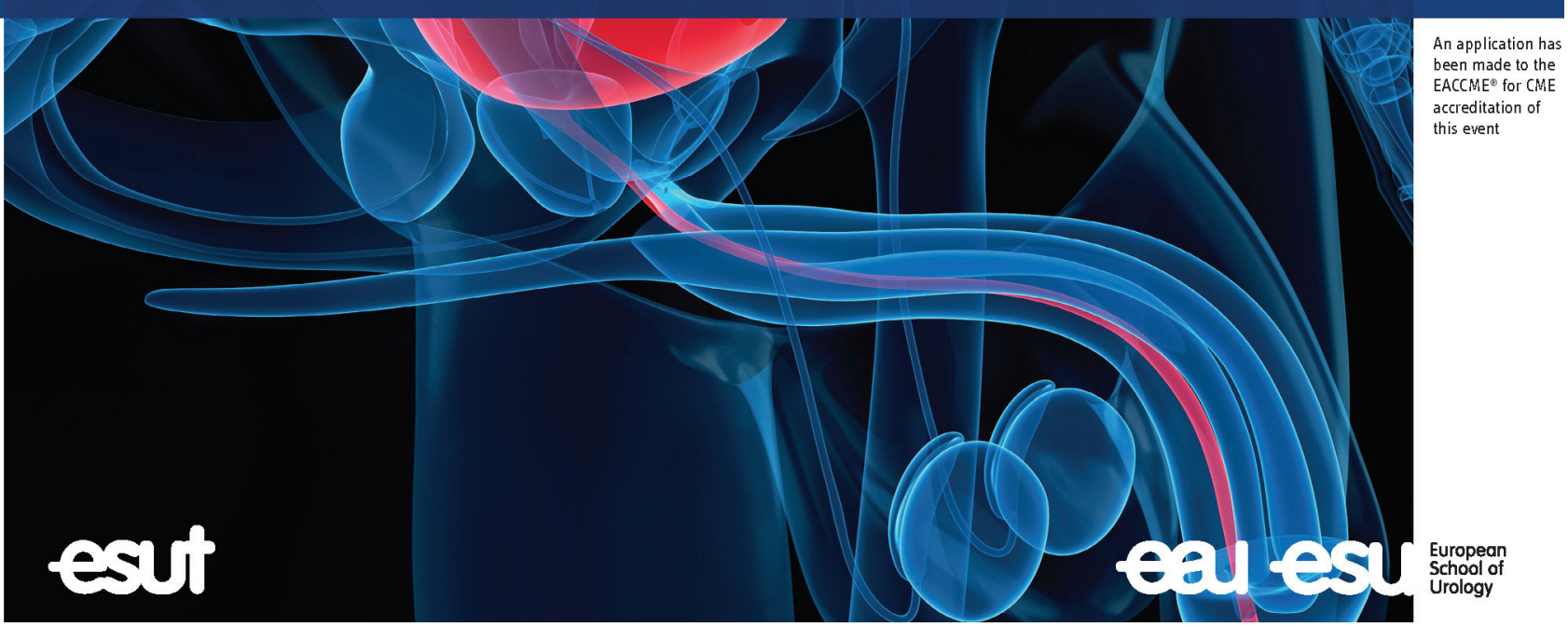

